\title{
Prospective randomized trial comparing sutured with sutureless mesh fixation for Lichtenstein hernia repair: long-term results
}

\author{
C. Kim-Fuchs $\cdot$ E. Angst $\cdot$ S. Vorburger $\cdot$ \\ C. Helbling $\cdot$ D. Candinas $\cdot$ R. Schlumpf
}

Received: 6 January 2011 / Accepted: 1 July 2011 / Published online: 26 July 2011

(C) Springer-Verlag 2011

\begin{abstract}
Background Following Lichtenstein hernia repair, up to $25 \%$ of patients experience prolonged postoperative and chronic pain as well as discomfort in the groin. One of the underlying causes of these complaints are the compression or irritation of nerves by the sutures used to fixate the mesh. We compared the level and rate of chronic pain in patients operated with the classical Lichtenstein technique fixated by sutures to patients with sutureless mesh fixation technique.

Methods A two-armed randomized trial with 264 male patients was performed. After consent, patients were randomized preoperatively. For the fixation of the mesh we used either sutures with slow-absorbing material (PDS 2.0) (group I, $n=133$ ) or tissue glue (Histoacryl) (group II, $n=131$ ). Follow-up examinations were performed after 3 , 12 months and after 5 years.

Results Patient characteristics in the two groups were similar. No cross-over between groups was observed. After 5 years, long-term follow-up could be completed for $59 \%$ of subjects. After 5 years, 10/85 (11.7\%) patients in group I and $3 / 70(4.2 \%)$ in group II suffered from chronic pain in the groin region $(P=0.108)$. The operation time was significantly shorter in group II (79 $\mathrm{min}$ vs $73 \mathrm{~min}, P=0.01$ ). One early recurrence occurred in group II (3 months). The recurrence rate was 0 and $0 \%$ after 12 months and 5.9\%
\end{abstract}

C. Kim-Fuchs · C. Helbling · R. Schlumpf

Clinic of Surgery, Kantonsspital Aarau, Aarau, Switzerland

C. Kim-Fuchs $(\varangle) \cdot$ E. Angst $\cdot$ S. Vorburger $\cdot$ D. Candinas University Clinic of Visceral Surgery and Medicine, Inselspital, University of Bern, 3010 Bern, Switzerland

e-mail: Corina.kim@insel.ch
$(5 / 85)$ and $10 \%(7 / 70)$ after 5 years in group I and group II, respectively $(P=0.379)$.

Conclusion After 5 years, the two techniques of mesh fixation resulted in similar rates of chronic pain. Whereas recurrence rates were comparable, fixation of the mesh with tissue glue decreased operating room time significantly. Hence, suture less mesh fixation with Histoacryl is a sensible alternative to suture fixation and should be especially considered for patients prone to pain.

Keywords Inguinal hernia $\cdot$ Lichtenstein hernia repair . Suture free mesh $\cdot$ Tissue glue $\cdot$ Chronic pain $\cdot$ Recurrence

\section{Introduction}

The Lichtenstein hernia repair is a common surgical procedure worldwide. First described in 1989, it has changed hernia surgery drastically $[1,2]$. The implantation of a mesh graft to stabilize the transverse fascia according to Lichtenstein is an operation of low complexity and is associated with a low rate of complications and recurrences [2-4]. Because the operation can be performed under local anesthesia it is well suited to outpatient surgery. Nevertheless, the rare occurrence of chronic pain after Lichtenstein hernia repair was recognized as an issue in the late 1980s [5]. In the 1990s, several authors published studies finding up to $50 \%$ of patients having postoperative groin discomfort [5-10]. The main causes identified as contributing to groin discomfort include: inflammatory reaction to the mesh, nerve lesion or nerve irritation and/or nerve entrapment by fixative sutures [6]. Because hernia repair with or without mesh implantation resulted in similar rates of chronic groin discomfort, suture-induced irritation of nerves is considered a major underlying factor. This is why 
alternative, sutureless mesh fixation techniques are of major interest: to date only preliminary data for the use of skin staples (rotating skin stapler) [11], spiral tacks [12] or tissue adhesives [13-17] have been published [11-17]. Because direct nerve irritation or nerve entrapment is eliminated, mesh fixation with glue (tissue adhesive) seems an optimal choice to reduce postoperative pain. Accordingly, preliminary results published with different glues all showed promising results with reduced postoperative pain [13-19]. In line with these results, we previously reported on short-term outcomes after mesh fixation with glue [20]. However, because glue is absorbed after a short time, the question remained whether this atraumatic fixation could lead to increased rates of recurrence. This study aimed to evaluate the rate of postoperative, chronic pain in patients operated on for inguinal hernia with the classic Lichtenstein technique (using suture fixation) or with glue fixation.

Glue has to meet the following criteria: biocompatibility, fast polymerization so it does not prolong operation time, and ideally inexpensive - at least the total operative costs should be comparable to 'traditional' suture fixation. In addition, application should be easy to learn and pack size should be adequate for the fixation of one mesh. Two different glues fulfilling all these requirements are available on the market: n-butyl-cyanoacrylat and human fibrin glue. Both are used as tissue adhesive in different applications. Another study comparing $\mathrm{N}$-butyl-2-cyanoacrylate and human fibrin glue for mesh fixation in primary inguinal hernia repair in a single-surgeon randomized trial sutures found no significant difference between these two glues [21]. Therefore, in this study to evaluate postoperative pain with and without suture fixation of the mesh implant, we chose Histoacryl $^{\circledR}$ (n-butyl-cyanoacrylat) because it is less expensive than human fibrin glue. In a first publication we reported the feasibility and low morbidity of mesh fixation with this tissue adhesive after the first 46 patients were followed for 3 months [20]. The primary endpoint of the study was postoperative chronic pain. Recurrence rates after 5 years were the secondary outcome parameter. Here, we show the long-term results after a 5-year follow-up.

\section{Patients and methods}

Between January 2001 and December 2004, 264 male patients with a preoperative diagnosis of inguinal hernia were recruited to the trial at the Kantonspital Aarau, Switzerland after giving informed consent. Hernias were classified according to Schumpelick by localization ( $\mathrm{L}=$ lateral, $\mathrm{M}=$ medial and $\mathrm{ML}=$ combined medial and lateral defect) and by size (I $<1.5 \mathrm{~cm}$, II $1.5-3 \mathrm{~cm}$ and III $>3 \mathrm{~cm}$ ) [23]. The inclusion criteria were: male, age over 25 years, primary unilateral hernia, elective surgery, and hernias
Table 1 Inclusion and exclusion criteria

\begin{tabular}{ll}
\hline Inclusion criteria & Exclusion criteria \\
\hline Age $>25$ years & Age $<25$ years \\
Elective operation & Emergency \\
Primary hernia & Recurrent hernia \\
Inguinal hernia & Femoral hernia \\
Size of defect (L III, & Size of defect (L I, L II, M I) \\
M II, M III, ML) & Hydrocele or varicocele on hernia side \\
& Infected operation field \\
& Immune deficiency \\
\hline
\end{tabular}

classified as LIII, MII, MIII and ML (Table 1). Patients were randomized by numbered sealed envelopes: the envelope was opened intraoperatively after the hernia was classified. The trial was unblinded and data were collected prospectively. The trial was approved by the local and regional ethic committee and registered with the Swiss federal office of public health (SFOPH-99-0021).

The following variables were evaluated: size of defect (hernia), type of anaesthesia, operation time, experience of the surgeon, resection of the nerves (ilioinguinal, genitofemoral), hospitalization time, risk factors, complications, recurrences, pain and hypesthesia and patient satisfaction. The primary endpoint of the study was postoperative chronic pain in the groin (painful fixation-sites). Chronic pain was defined as persistent discomfort or pain in the groin for at least 3 month postoperatively [22]. The secondary endpoints were recurrences (defined as clinical recurrences), hypesthesia and postoperative morbidity.

Follow-up examinations were at 3 and 12 months as well as after 5 years. They included an interview with questionnaire and clinical examination by a senior physician. After 5 years, patient satisfaction was evaluated by a subjective patient scoring from 1 to $10 \quad(1=$ not satisfied at all, $10=$ completely satisfied). In the case of recurrence and reoperation, the patients were excluded from further follow-up.

\section{Surgical technique}

The surgical technique was as described previously [20]. Briefly, local anaesthesia with or without sedation was preferred, spinal or general anaesthesia were used alternatively. After dissection of the inguinal region, the hernias were graded according to Schumpelick classification [23]. Only patients with LIII, M II-III, or ML hernia were included in the trial, the remaining patients were treated with a Shouldice hernia repair. The mesh was a VIPRO II (Ethicon, Johnson \& Johnson Medical Products, Vienna , Austria) fixed by PDS 2.0 (polydioxanone; Ethicon) for the sutured technique (Group I) or Histoacryl ${ }^{\circledR}$ (Braun Medical, Sempach, Switzerland) for the sutureless technique (Group 
II). In group I, the mesh was fixed to the aponeurotic tissue above the pubic tubercle along the inguinal ligament with a running suture and to the internal oblique muscle with interrupted sutures. Lateral to the internal ring, the mesh was incised and the overlying cranial part of the mesh was fixed to the lower part of the mesh and to the inguinal ligament with interrupted sutures. In group II, the positioning of the mesh was the same, but all sutures were replaced by small dots of Histoacryl ${ }^{\circledR}$. The operation was finished by closing the external aponeurosis with a Vicryl 2/0 running suture (polyglactin 910, Ethicon), subcutaneous drainage and an intracutaneous closure of the incision with Monosyn 5/0 (Glyconate, a terpolymer; Aesculap, Braun) in both groups. A senior surgeon supervised all operations performed by residents. As hernia repair is one of the primary teaching operations, the performing residents were in the 2nd or higher year of education. The postoperative procedure was identical in both groups. The patients moved freely but lifting was restricted to $7 \mathrm{~kg}$ for the first 2 weeks.

\section{Statistical analysis}

Continuous data are presented as mean \pm one standard deviation (1 SD) and a $t$ test was used where appropriate.
Proportions were compared with Chi-squared or Fisher's exact test. Duration of pain and the occurrence of recurrences was analysed the Kaplan-Meier method with the log-rank test. An alpha value below 0.05 was determined as significant. All statistics were performed with the statistical software, SPSS Statistics version 17.0 (SPSS, Chicago, IL).

\section{Results}

A total of 264 male patients was included in the trial: 133 (50.4\%) patients were allocated to the sutured mesh fixation (group I) and $131(49.6 \%)$ patients to the sutureless mesh fixation (group II). Patient characteristics are detailed in Table 2. The groups did not differ in type of hernia, age, anaesthesia or experience of the surgeon. The operation time was significantly shorter in group II with the sutureless mesh (79 vs $73 \mathrm{~min}$ ). Mean hospital stay was 3.4 days in both groups (Table 3). After 3 months, 2 patients in each group were lost to follow-up, after 12 months 13 in group I and 17 in group II. After 5 years, we again lost 33 patients in group I and 41 in group II (Fig. 1).

Table 2 Patient characteristics

\footnotetext{
${ }^{\text {a }}$ Values are median (range)

b Values in parentheses are percentages

c Fisher's exact test

d Student's $t$ test
}

Table 3 Postoperative results

\footnotetext{
${ }^{\text {a }}$ Values are median (range)

b Values in parentheses are percentages

c Student's $t$ test
}

\begin{tabular}{|c|c|c|c|c|}
\hline & Group I (sutured) & Group II (sutureless) & Total & $P$ \\
\hline No. of patients ${ }^{b}$ & $133(50.4)$ & $131(49.6)$ & $264(100)$ & \\
\hline Age $^{\mathrm{a}}$ [years] & $56.8(25-83)$ & $55.1(28-85)$ & $56.1(25-85)$ & $0.378^{\mathrm{d}}$ \\
\hline \multicolumn{5}{|l|}{ Type of hernia ${ }^{b}$} \\
\hline LIII & $45(33.8)$ & $41(31.1)$ & $86(32.6)$ & 0.695 \\
\hline MII & $12(9.1)$ & $7(5.2)$ & $19(7.2)$ & $0.341^{\mathrm{c}}$ \\
\hline MIII & $32(24.1)$ & $39(29.7)$ & $71(26.9)$ & $0.332^{\mathrm{c}}$ \\
\hline MLII & $9(6.7)$ & $4(3.0)$ & $13(4.9)$ & $0.255^{\mathrm{c}}$ \\
\hline MLIII & $35(26.3)$ & $40(31)$ & $75(28.4)$ & $0.412^{\mathrm{c}}$ \\
\hline \multicolumn{5}{|l|}{ Anaesthesia ${ }^{b}$} \\
\hline Local & $113(84.2)$ & $106(80.9)$ & $219(83.3)$ & $0.416^{\mathrm{c}}$ \\
\hline Spinal/epidural & $8(6.0)$ & $5(3.8)$ & $13(4.9)$ & $0.571^{\mathrm{c}}$ \\
\hline General & $12(9.1)$ & $20(15.3)$ & $32(12.1)$ & $0.134^{\mathrm{c}}$ \\
\hline \multicolumn{5}{|l|}{ Surgeon ${ }^{\mathrm{b}}$} \\
\hline Resident & $109(82)$ & $109(83.2)$ & $218(82.6)$ & $0.871^{\mathrm{c}}$ \\
\hline Staff surgeon & $5(3.8)$ & $3(2.3)$ & $8(3.0)$ & $0.722^{\mathrm{c}}$ \\
\hline Chief physician & $19(14.2)$ & $19(14.5)$ & $38(14.4)$ & $1.0^{\mathrm{c}}$ \\
\hline \multirow[t]{2}{*}{ Mean operative time $(\min )$} & $79(40-120)$ & $73(35-150)$ & $76.3(35-150)$ & $0.01^{\mathrm{d}}$ \\
\hline & $\begin{array}{l}\text { Group I } \\
\text { (sutured) } n=133\end{array}$ & $\begin{array}{l}\text { Group II } \\
\text { (sutureless) } n=131\end{array}$ & Total $n=264$ & $P$ \\
\hline Major complication $^{\mathrm{b}}$ & 0 & 0 & 0 & \\
\hline Hematoma $^{\mathrm{b}}$ & $5(3.7)$ & $3(2.3)$ & $8(3)$ & ns. \\
\hline Reoperations & 0 & 0 & 0 & \\
\hline Hospital stay ${ }^{\mathrm{a}}$ & $3.39(1-9)$ & $3.35(2-13)$ & $3.37(1-13)$ & $0.816^{\mathrm{c}}$ \\
\hline
\end{tabular}


Fig. 1 Flow chart of randomized trial

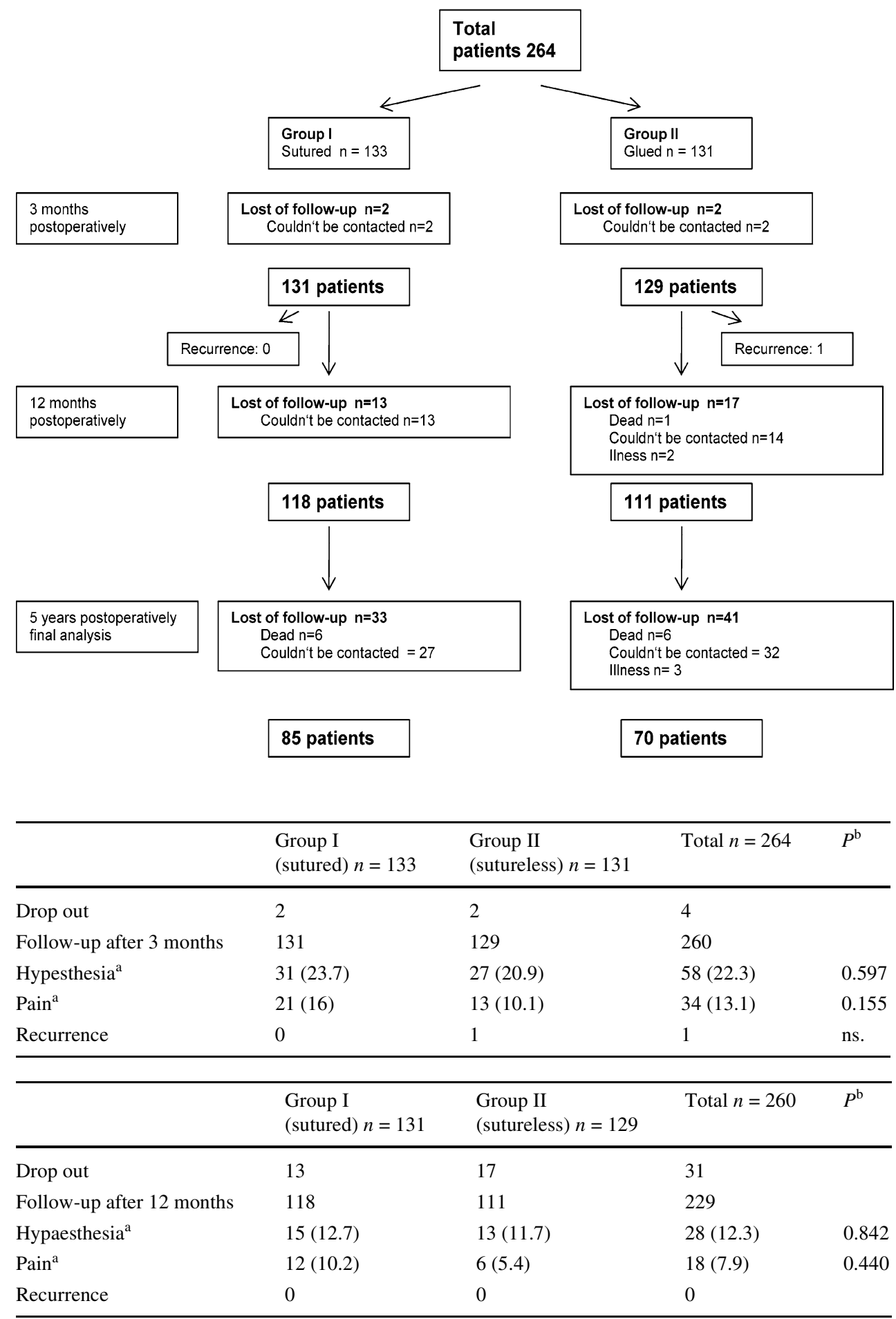

Table 4 Follow-up after 3 months

\footnotetext{
a Values in parentheses are percentages

${ }^{\mathrm{b}}$ Fisher's exact test
}

Table 5 Follow-up after 12 months

a Values in parentheses are percentages

${ }^{\mathrm{b}}$ Fisher's exact test
$12 / 118(10.2 \%)$ in group I and $13 / 129(10.1 \%)$ to $6 /$ $111(5.4 \%)$ in group II (Tables 4,5$)$. After 5 years, we found $10 / 85(12.2 \%)$ patients in group I and $3 / 70(4.3 \%)$ in group II to suffer from persistent pain or discomfort in the groin region $(P=0.44)$. All patients with persistent pain qualified the pain as being "mild", with no loss of quality of life and no interference with daily activity (Table 6). 
Table 6 Follow-up after 5 years

${ }^{\text {a }}$ Values in parentheses are percentages

${ }^{\text {b }}$ Fisher's exact test

\begin{tabular}{lllll}
\hline & $\begin{array}{l}\text { Group I } \\
\text { (sutured) } n=118\end{array}$ & $\begin{array}{l}\text { Group II } \\
\text { (sutureless) } n=111\end{array}$ & Total $n=229$ & $P^{\mathrm{b}}$ \\
\hline Drop out & 33 & 41 & 74 \\
Follow-up after 5 years $^{\text {Hypesthesia }}{ }^{\mathrm{a}}$ & 85 & 70 & 155 & \\
Pain $^{\mathrm{a}}$ & $11(13.4)$ & $9(12.8)$ & $20(12.9)$ & 1.0 \\
Recurrences $^{\mathrm{a}}$ & $10(12.2)$ & $3(4.2)$ & $13(8.4)$ & 0.108 \\
Overall recurrences & $5 / 85(5.8)$ & $7 / 70(10)$ & $12 / 155(7.7)$ & 0.379 \\
\hline
\end{tabular}

The ilioinguinal nerve was resected in nine patients in both groups. The genital branch of the genitofemoral nerve was resected in seven and eight patients in group I and II, respectively. For patients with persistent pain in group I, the nerves were preserved in eight out of ten cases, in one case the surgeon resected the ilioinguinal nerve and in one case the genitofemoral nerve. In group II, both nerves were identified and preserved during surgery for all patients. In contrast, seven and six pain-free patients of group I had a resection of the ilioinguinal nerve or the genitofemoral nerve, respectively. In group II, pain-free patients had nine and eight nerve resections. Persistent pain was independent of surgical nerve resection.

Secondary endpoints: recurrence, hypesthesia, morbidity

At 3 months follow-up, there was one recurrence in group II, with none at 12 months follow-up. At the 5-year follow-up, we found $5 / 85(5.8 \%)$ recurrences in group I and 7/70 (10\%) in group II $(P=0.379)$. At 3 months, 31 patients from group I $(23.7 \%)$ recorded hypesthesia in the groin region, compared to 27 patients $(20.9 \%)$ in group II $(P=0.155)$. At 12 months, the number of patients with hypesthesia diminished from $31 /$ $131(23.7 \%)$ to $15 / 118(12.7 \%)$ in group I, and from $27 / 129$ $(20.9 \%)$ to $13 / 111(11.7 \%)$ in group II (Tables 4 and 5). After 5 years, 11/85 (13.4\%) and 9/70 (12.9\%) patients recorded hypesthesia in the groin region for group I and group II, respectively. There was no correlation between hypesthesia and nerve preservation.

No major complication, no reoperation and no infection of the mesh occurred in either group. We found five minor complications (hematoma) in group I and three in group II (ns). Patients of both groups were very satisfied with the postoperative outcome. Patients from group I scored their satisfaction with an average of 9.21 (range 5-10), patients from group II had an average of 9.45 (range 6-10). There was no significant difference $(P=0.167)$.

\section{Discussion}

This prospective randomized study compared mesh fixation with sutures to sutureless mesh fixation with glue in Lich- tenstein hernia repair. It provides the first evidence that fixation with glue is not inferior to the standard sutured technique on long-term outcomes. Our results suggest a trend toward less pain and more recurrences for the sutureless technique. Therefore Lichtenstein hernia repair with sutureless mesh fixation is a valid alternative to fixation by sutures, especially in patients prone to pain.

The primary endpoint of the trial was the presence of chronic pain in the groin, defined as persistent pain after 3 or more months. Although Lichtenstein hernia repair is associated with low morbidity, several authors have reported high rates of patients with chronic pain after at least 3 months [5, 24-26]. A large randomized multicenter trial from Sweden [29] has shown $24.8 \%$ of patients with chronic pain after 1 year; at 5-year follow-up, the authors found $18.8 \%$ of the patients with groin discomfort, and $3.5 \%$ with moderate or severe pain. Although the Lichtenstein hernia repair is a tension-free method, pain may originate from nerve resection, a periostal reaction, tension on muscle fibers, nerve compression due to the sutures, or a foreign-body reaction caused by the mesh itself [14]. One method to reduce postoperative pain was thought to be the use of a sutureless technique.

In group I patients, we found chronic pain in $21 / 131$ (16\%) patients after 3 months and the number diminished to $12 / 118(10.2 \%)$ after 12 months. In group II, after 3 months $13 / 129(10.1 \%)$ had pain and 6/129 (5.4\%) after 12 months. At the 5-year follow-up the trend continued, with chronic pain in 10/85 (12.2\%) for the sutured and $3 / 70$ $(4.2 \%)$ for the sutureless group. The increase in pain in the sutured group is not due to an absolute increase, but rather to the dropout of patients. The differences between group I and II are not significant, but they show a trend toward less pain in the sutureless group. This is in accordance with studies published by Hidalgo et al. [14] and Nowosbilski et al. [17] showing less pain after sutureless Lichtenstein hernia repair compared to the sutured technique. Nevertheless, in these latter studies, the results were also not significant and no long-term follow-up is available. We show here that the trend for less pain in the sutureless group persists after 5 years of follow-up. We found no effect of nerve preservation versus division on chronic pain and hypesthesia. This is in accordance with results found in other studies 
$[5,6]$, and suggests that the problem of chronic pain is not due to topical nerve preservation or division, but rather due to the mesh or mesh fixation.

As secondary endpoints we analyzed the recurrence rate, hypesthesia and morbidity. We found one recurrence in the sutureless group at 3 months follow-up. After such a short time the reason for a recurrence is likely to be a technical problem and not the sutureless technique per se. After 1 year we did not find any more recurrences, resulting in a 1 -year recurrence rate of $0 \%(0 / 118)$ in the sutured, and $0.9 \%(1 / 111)$ in the sutureless group. This is in accordance with other studies using tissue adhesives that showed no recurrences after 1 year of follow-up [13, 14, 16, 17, 29]. At the 5-year follow-up, we found 5.8\% (5/85) in the sutured and $10 \%(7 / 70)$ in the sutureless group. In our study, $82 \%$ of all operations were performed by residents. A senior surgeon supervised all these operations. Although these rates are higher than the 1.0-4.9\% reported by general surgeons, especially in the sutureless group [27-30], we believe that, in a teaching hospital setting, where mainly residents perform this surgery following a standard proto$\mathrm{col}$, these rates are acceptable. Again the differences are not significant, but show a trend towards more recurrences for the sutureless technique. These long-term results are unique and show that, after a 5-year follow-up, there is a trend towards more recurrences with the sutureless technique. There was a decrease in hypesthesia after 12 months in both groups, and a relative increase after 5 years due to the dropout of patients. These rates are comparable to those in the literature $[5,18,19]$.

A critical point of this clinical trial is the loss of patients to follow up (110 of 264) over 5 years. We used stringent criteria for inclusion. Only patients seen in the office with a clinical examination were included and patients doing well but not willing to be examined were excluded. Of the rest, 13 patients died during the 5-year period, 5 had a illness, which prevented them from coming to the hospital, 34 patients moved and could not be contacted and 58 were not willing to be examined again. The satisfaction with the operation result was equal in both groups. Thus we do not believe that the dissatisfaction was the reason for the greater drop out rate in group II.

Lichtenstein hernia repair is well known, safe, easy to teach and has a low morbidity and mortality rate [2-4]. Nevertheless, several authors have recently published high percentages of postoperative discomfort and chronic pain [5-10]. We showed in this study that a modified sutureless alternative is not inferior to the sutured technique. The sutureless technique tends to result in less pain, but higher recurrence rates. Therefore it is sensible to use the sutureless technique for patients prone to pain. In the current economic setting it is important to minimize pain to get patients back to work quickly, even at the price of a higher recurrence rate.

\section{References}

1. Lichtenstein IL (1989) The tension free hernioplasty. Am J Surg 157:188-193

2. Amid PK, Shulman AG, Lichtenstein IL (1994) A critical evaluation of the Lichtenstein tension-free hernioplasty. Int Surg 79:76-79

3. Amid PK, Lichtenstein IL (1998) Long-term results and current status of the Lichtenstein open tension-free hernioplasty. Hernia 2:89-94

4. Hetzer FH, Hotz T, Steinke W, Schlumpf R, Decurtins M, Largiader F (1999) Gold standard for inguinal hernia repair: shouldice or Lichtenstein? Hernia 3:117-120

5. Nienhuijs S, Staal E, Strobbe L, Rosman C, Groenewoud H, Bleichrodt R (2007) Chronic pain after mesh repair of inguinal hernia: a systematic review. Am J Surg 194:397-400

6. Paajanen H (2002) Do absorbable mesh sutures cause less chronic pain than nonabsorbable sutures after Lichtenstein inguinal herniorrhaphy? Hernia 6:26-28

7. The MRC Laparoscopic Groin Hernia Trial Group (1999) Laparoscopic versus open repair of groin hernia: a randomised comparison. Lancet 354:185-190

8. Starling JR, Harms BA, Schroeder ME (1987) Diagnosis and treatment of genitofemoral and ilioinguinal entrapment neuralgia. Surg 102:581-586

9. Cunningham J, Temple WJ, Mitchell P (1996) Cooperative hernia study: pain in the postrepair patient. Ann Surg 224:598-602

10. Gillion JF, Fagniez PL (1999) Chronic pain and cutaneous sensory changes after inguinal hernia repair: comparison between open and laparoscopic techniques. Hernia 3:75-80

11. Mills IW, McDermott IM, Ratliff DA (1998) Prospective randomized controlled trial to compare skin staples and polyproylene for securing the mesh in inguinal hernia repair. Br J Surg 85:790-792

12. Douglas JM, Young WN, Jones DB (2002) Lichtenstein inguinal herniorraphy using sutures versus tacks. Hernia 6:99-101

13. Farouk R, Drew PJ, Quershi A, Roberts AC, Duthie GS, Monson JRT (1996) Preliminary experience with butyl-2-cyanoacrylate adhesive in tension-free inguinal hernia repair. Br J Surg 83:1100

14. Nowobilski W, Dobosz M, Wojciechowicz T, Mionskowska L (2004) Lichtenstein inguinal hernioplasty using Bytyl-2Cyanoacrylate versus sutures. Eur Surg Res 36:367-370

15. Jourdan IC, Bailey ME (1998) Initial experience with the use of n-butyl-cyanoacrylate glue for the fixation of polypropylene mesh in laparoscopic hernia repair. Surg Laparosc Endosc 8:291-293

16. Canonico S, Santoriello A, Campitiello F, Fattopace A, Della Corte A, Sordelli I, Benevento R (2005) Mesh fixation with human fibrin glue (Tissucol) in open tension-free inguinal hernia repair: a preliminary report. Hernia 9:330-333

17. Hidalgo M, Castillo M, Eymar J, Hidalgo A (2005) Lichtenstein inguinal hernioplasty: sutures versus glue. Hernia 9:242-244

18. Canonico S, Benevento R, Della Corte A, Fattopace A, Canonico $R$ (2007) Sutureless tension-free hernia repair with human fibrin glue (Tissucol) in soccer players with chronic inguinal pain: initial experience. Int J Sports Med 28:873-876

19. Campanelli G, Champault G, Hildalgo Pascual M (2008) Randomized, controlled, blinded trial of Tissucol/Tisseel for mesh fixation in patients undergoing Lichtenstein technique for primary inguinal hernia repair: rationale and study design of the TIMELI trial. Hernia 12:159-165 
20. Helbling C, Schlumpf R (2003) Sutureless Lichtenstein: first results of a prospective randomized clinical trial. Hernia 7:80-84

21. Testini M, Lissidini G, Poli E et al (2010) A single-surgeon randomized trial comparing sutures, N-butyl-2-cyanoacrylate and human fibrin glue for mesh fixation during primary inguinal hernia repair. Can J Surg 53:155-160

22. Merskey HBN (1994) Classification of chronic pain, descriptors of chronic pain syndromes and definitions of pain terms, 2 nd edn. IASP, Seattle

23. Schumpelick V, Treutner KH, Arlt G (1994) Klassifikation von Inguinalhernien. Chirurgie 65:877-879

24. Butters M, Redecke J, Köninger J (2007) Long-term results of a randomized clinical trial of Shouldice, Lichtenstein and transabdominal preperitoneal hernia repair. Br J Surg 94:562-565

25. Frey D, Wildisen A, Hamel C, Zuber M, Oertli D, Metzger J (2007) Randomized clinical trial of Lichtenstein's operation versus mesh plug for inguinal hernia repair. Br J Surg 94:36-41
26. Callesen T, Bech K, Kehlet H (1999) Prospective study of chronic pain after groin hernia repair. Br J Surg 86:1528-1531

27. Hasegawa S, Yoshikawa T, Yamamoto Y, Ishiwa N, Morinaga S, Noguchi Y, Ito H, Wada N, Inui K, Imada T, Rino Y, Takanashi Y (2006) Long-term outcome after hernia repair with the prolene hernia system. Surg Today 36(12):1058-1062

28. Sanjay P, Harris D, Jones P, Woodward A (2006) Randomized controlled trial comparing prolene hernia system and Lichtenstein method for inguinal hernia repair. ANZ J Surg 76(7):548-552

29. Eklund A, Montgomery A, Bergkvist L, Rudberg C (2010) Swedish multicentre trial of inguinal hernia repair by laparoscopy (SMIL) study group. Chronic pain 5 years after randomized comparison of laparoscopic and Lichtenstein inguinal hernia repair. Br J Surg 97(4):600-608

30. Benizri EI, Rahili A, Avallone S, Balestro C, Cai J, Benchimol D (2006) Open inguinal hernia repair by plug and patch: the value of fibrin sealant fixation. Hernia 10:389-394 